\title{
Causes of Failure of Anterior Cruciate Ligament Reconstruction and Revision Surgical Strategies
}

\author{
Paolo Di Benedetto, MD, PhD, Enrico Di Benedetto, MD, Andrea Fiocchi, MD, Alessandro Beltrame, MD, and \\ Araldo Causero, MD \\ Clinic of Orthopaedics, Academic Hospital of Udine, Udine, Italy
}

\begin{abstract}
Purpose: Long-term outcomes of anterior cruciate ligament (ACL) reconstruction are good or excellent; however, $0.7 \%-20 \%$ of patients suffer from recurrent instability due to graft failure. The purpose of this paper was to analyse failure aetiology and the possibilities of revision surgical strategies, with a description of our experience. We obtained optimal and good results in most of our patients.

Materials and Methods: We retrospectively reviewed 42 patients who underwent revision surgery (43 revisions) due to relapsing instability after ACL reconstruction between 2006 and 2015. We used allografts in 39 cases and autografts in 4 cases.

Results: The $85.7 \%$ of the patients obtained optimal results (normal knee; group A) and the $7.2 \%$ obtained good results (nearly normal knee; group B) according to the International Knee Documentation Committee score. The most frequent failure causes were traumatic events, non-anatomic tunnel placement, and lack of graft incorporation.

Conclusions: A correct revision surgery requires accurate patient evaluation and knee imaging. Preoperative planning starts with the identification of the cause of failure of the primary reconstruction. Then, the most suitable procedure should be determined for each case. It is also important to accurately inform the patient of all the complexity of an ACL revision surgery even if it is a procedure with high rates of excellent and good outcomes.
\end{abstract}

Keywords: Knee, Anterior cruciate ligament, Reconstruction, Failure, Revision

\section{Introduction}

There has been extensive agreement that ligament reconstruction is an optimal treatment strategy for functional instability of the knee caused by anterior cruciate ligament (ACL) deficiency ${ }^{1-4)}$. Long-term outcomes of ACL reconstruction are good or excellent in terms of restoration of articular stability, improvement of symptoms, and return to preinjury activities (range, 75\% to $97 \%)^{1-3,5,6)}$. Unfortunately, $0.7 \%-20 \%$ of patients experience

Received January 22, 2016; Revised (1st) May 6, 2016;

(2nd) June 13, 2016; (3rd) July 26, 2016; Accepted July 29, 2016

Correspondence to: Paolo Di Benedetto, MD, PhD

Clinic of Orthopaedics, Academic Hospital of Udine, Piazzale Santa

Maria della Misericordia 15, 33100 Udine, Italy

Tel: +39-043-255-9464, Fax: +39-043-255-9298

E-mail:paodibenedetto@gmail.com

This is an Open Access article distributed under the terms of the Creative Commons Attribution Non-Commercial License (http://creativecommons.org/licenses/by-nc/4.0/) which permits unrestricted non-commercial use, distribution, and reproduction in any medium, provided the original work is properly cited. recurrent instability due to graft failure ${ }^{4,-11)}$. Patients have a $5.4 \%$ risk of undergoing a revision surgery within five years after primary ACL reconstruciton ${ }^{6}$. In the USA, the annual incidence of ACL tears is approximately 1 in 3,000 people: more than 175,000 people had an ACL injury every year and about 100,000 operations were performed every year in the last decades ${ }^{1-3,5,6,12-14)}$. Considering the progressive increase in ACL injury and the need for reconstruction surgery ${ }^{1,25)}$, it is reasonable to expect that the incidence of failure will also increase. A successful ACL revision surgery requires a methodical approach to identification and correction of all potential causes of failure. Thus, it is extremely important to make an accurate preoperative plan based on anamnesis and clinical and radiological evaluations. Several factors can influence the success of revision surgery including the preinjury laxity and integrity of secondary stabilizers; articular cartilage and meniscus status, graft type, surgical technique, rehabilitation, and patient's compliance, enthusiasm, and expectation ${ }^{8)}$.

Accurate identification of the cause of failure is needed to select the most appropriate revision strategy for each patient. For example, the operation can be carried out in one or two sessions 
depending on the case. If a failure is only due to incorrect femoral tunnel placement, a revision surgery can be done in a single session. In other circumstances, there is a need for two different surgical sessions, as in the case of tunnel enlargement with massive bone stock decrease.

In this study, we analysed failure aetiology and possibilities of revision surgical strategies with a description of our experience in $\mathrm{ACL}$ revision surgery.

\section{Materials and Methods}

Between 2006 and 2015, we observed 47 patients with relapsing instability after ACL reconstruction. Five patients decided not to subject themselves to ACL revision surgery, so they were excluded from the study. The remaining 42 patients underwent ACL revision. Since one patient underwent surgery twice, a total of 43 ACL revisions were reviewed. All surgeries were performed by one senior surgeon. The same rehabilitation program was applied to all patients. There were 9 females and 33 males. Their

Table 1. Demographic Data and Follow-up

\begin{tabular}{lc}
\hline \multicolumn{1}{c}{ Variable } & Mean \pm SD \\
\hline Sex (M:F) & $33: 9$ \\
Age at revision ACL reconstruction (yr) & $32.6 \pm 10.18$ \\
Average time between 1st ACL recosntruction and & $2.5 \pm 2.48$ \\
ACL revision (yr) & \\
Follow-up from revision ACL reconstruction (mo) & $42.7 \pm 27.1$ \\
\hline
\end{tabular}

SD: standard deviation, ACL: Anterior cruciate ligament.

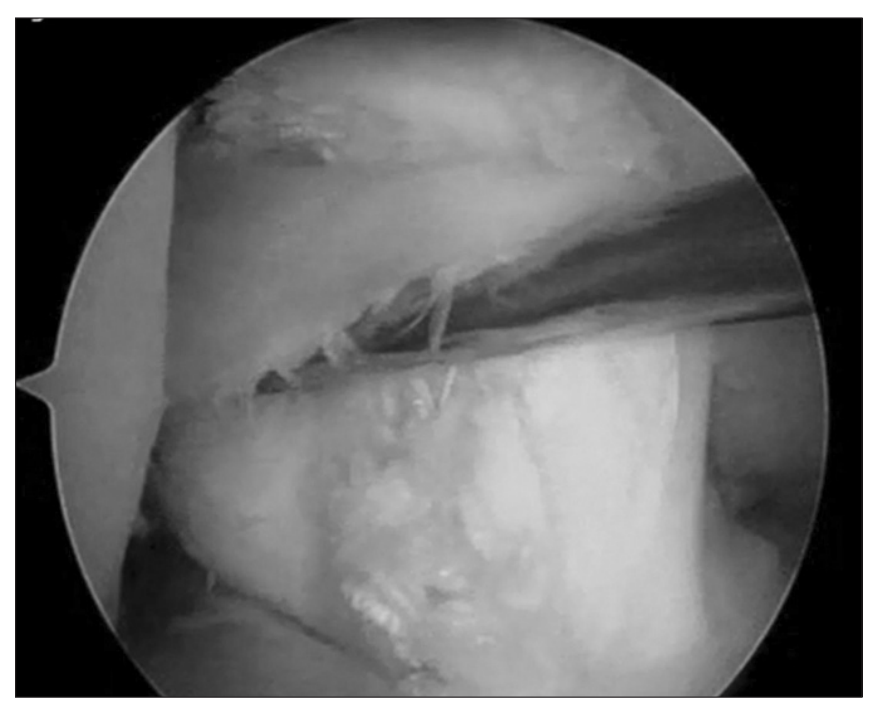

Fig. 1. Arthroscopic view of biological failure after anterior cruciate ligament reconstruction with allograft (cryopreserved patellar tendon). average age was $32.6 \pm 10.18$ years (range, 16 to 53.6 years). The average time elapsed from the first ACL reconstruction was about $2.5 \pm 2.48$ years (range, 9 months to 14 years) (Table 1 ).

All patients were evaluated clinically and radiologically with magnetic resonance imaging and radiography. Furthermore, possible associated instabilities of the joint were investigated under anaesthesia during revision surgery. Anterior shifting of the operated knee was compared to that of the contralateral knee using an arthrometer (Rolimeter; Aircast Europa, Neubeuern, Germany). In the first ACL reconstruction, autologous bone-patellar tendonbone (BPTB) were used in 27 patients, gracilis and semitendinosus tendons with top traction system (TTS; Orthoplus s.r.l., Padua, Italy) technique in 11 patients, cryopreserved patellar tendon allograft in 2 patients (Fig. 1), and synthetic ligaments (Dacron; Meadox Medicals, Oakland, NJ, USA) in 3 patients (Table 2).

After revision surgery, the average follow-up was $42.7 \pm 27.1$ months (range, 10 to 108 months). All patients were evaluated using the International Knee Documentation Committee (IKDC) scale, Lysholm score, and Tegner activity scale.

\section{Results}

The results (both subjective and objective) of the revision surgery using the IKDC scale were as follows: 36 patients $(85.7 \%)$ obtained optimal results (normal knee; group A), 3 patient $(7.2 \%)$ obtained good results (nearly normal knee; group B), 2 patients (4.8\%) obtained unsatisfactory results (abnormal knee; group C), 1 patient (2.4\%) obtained bad results (seriously abnormal knee; group D). Thus, satisfactory results were obtained in $92.9 \%$ of the patients.

Subjective evaluation on the patient's general satisfaction using the IKDC score (Fig. 2) provided the following results: group A, 36 patients (85.6\%); group B, 3 patient (7.2\%); group C, 2 patients (4.8\%); and group D, 1 patient (2.4\%). Objective evaluation of the operated knee based on recovery of complete range of mo-

Table 2. Tendons Used in the First Anterior Cruciate Ligament Reconstruction

\begin{tabular}{lc}
\hline \multicolumn{1}{c}{ Variable } & $\begin{array}{c}\text { No.of } \\
\text { patients }\end{array}$ \\
\hline Autologous bone-patellar tendon-bone & 27 \\
Gracilis and semitendinosus tendons with TTS technique & 11 \\
Cryopreserved patellar tendon allograft & 2 \\
Synthetic ligament (Dacron) & 3 \\
Total & 43 \\
\hline
\end{tabular}

TTS: top traction system. 
tion, possible loss of extension and/or flexion, and positive laxity or articular instability tests (Jerk and Lachman tests) provided the following results: group A, 39 patients (92.8\%); group B, 0 patients (0\%); group C, 2 patients (4.8\%); and group D, 1 patients (2.4\%). Anteroposterior laxity evaluation was carried out with an arthrometer (Rolimeter), which yielded comparative values in relation to the contralateral knee between 0 and $2 \mathrm{~mm}$. With regard to the Lysholm score (Fig. 3), 39 patients obtained a score between 100 and 95 points, 1 patient had a score between 94 and 84 points, and 2 patients had a score between 83 and 65 points.

With regard to the Tegner activity level, 35 out of 42 patients

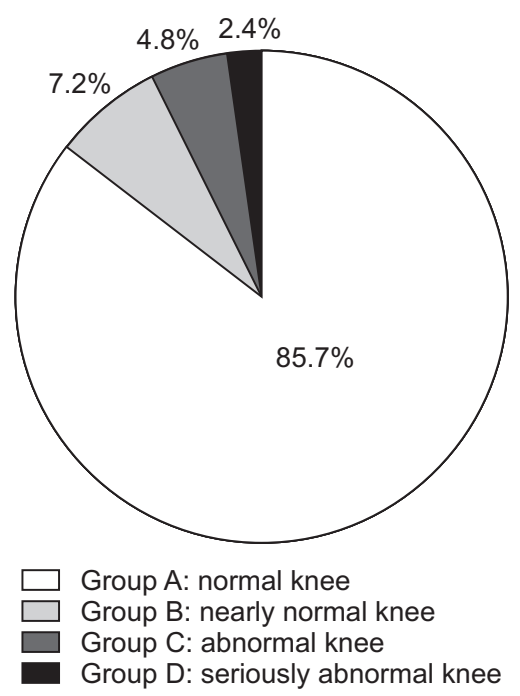

Fig. 2. International Knee Documentation Committee results: 36 patients $(85.7 \%)$ obtained optimal results (group A); 3 patients $(7.2 \%)$ obtained good results (group B); 2 patients (4.8\%) obtained unsatisfactory results (group C); 1 patient (2.4\%) obtained bad results (group D). Overall, good results (the first two classes) amounted to $92.9 \%$.

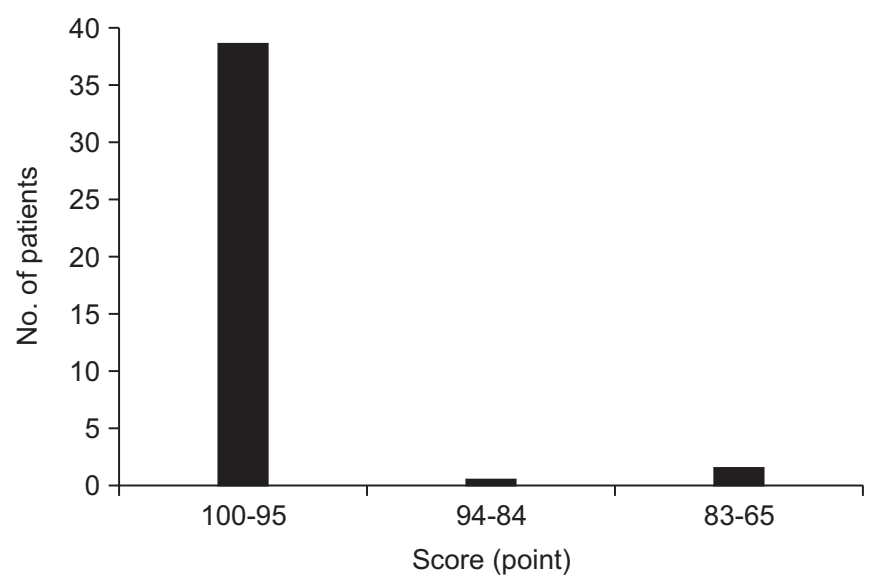

Fig. 3. Lysholm score results: 39 patients obtained a score between 100 and 95 points, 1 patient obtained a score between 94 and 84 points, 2 patients had a score between 83 and 65 points. had an average level of 6.70 increased from a preinjury average level of 6.58 at 12 months after surgery. Although no statistical significance was observed in this result, this indicates $83.3 \%$ of the patients obtained functional recovery that allowed sports activities they had performed before ACL failure. The Tegner level decreased from 7 to 3 in only 2 patients, and 5 patients decided to not to engage in previous activities for personal reasons. The majority of patients reported satisfaction with the operation and postoperative functional recovery. Some reported they felt insecure during sports activities, which often justified their assessment of "nearly normal knee" in spite of resumption of sports activities. Only 2 patients reported unsatisfied because they had not resumed preinjury level of activities due to pain, flexion deficit, or persistent giving way.

Regarding the failure aetiology, 32 out of 42 of the patients (76.2\%) had a traumatic sprain to the knee, whereas the others had progressive instability without an important trauma after primary ACL reconstruction. In particular, instability was found in 2 patients with synthetic ligaments and 8 patients (19.1\%) where anterior placement of the femoral tunnel (technical error) was noted during revision surgery.

Cryopreserved gracilis and semitendinosus tendon allografts (TTS technique) were used in 17 patients and cryopreserved patellar tendon allografts in 14 patients. We used autologous gracilis and semitendinosus tendons in 1 patient and autologous BPTB in 1 patient. We used cryopreserved anterior tibialis tendon allografts in 2 patients (Fig. 4) and cryopreserved Achilles tendon allografts in 2 patients. Cryopreserved semitendinosus tendon al-

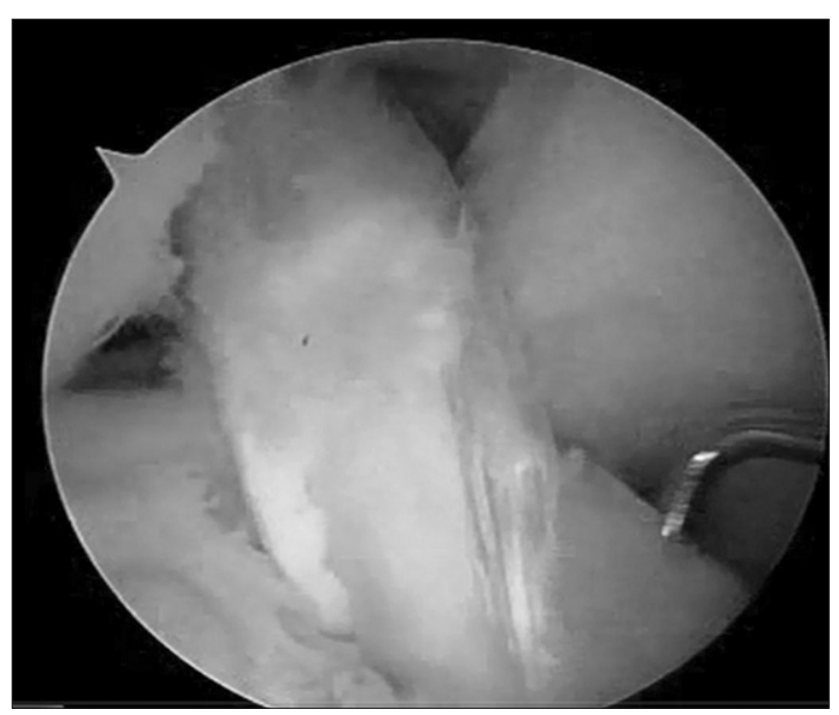

Fig. 4. Anterior cruciate ligament revision with the anterior tibialis tendon. 
Table 3. Tendons Used in the Anterior Cruciate Ligament Revision Surgery

\begin{tabular}{lc}
\hline \multicolumn{1}{c}{ Variable } & $\begin{array}{c}\text { No.of } \\
\text { patients }\end{array}$ \\
\hline $\begin{array}{l}\text { Cryopreserved gracilis and semitendinosus tendon } \\
\text { allografts (TTS technique) }\end{array}$ & 17 \\
Cryopreserved patellar tendon allograft & 14 \\
Autologous semitendinosus in 2 cases (TLS technique) & 4 \\
Cryopreserved semitendinosus tendon allograft & 2 \\
Cryopreserved anterior tibialis tendon allograft & 2 \\
Cryopreserved Achilles tendon allograft & 2 \\
Autologous gracilis and semitendinosus tendons & 1 \\
Autologous bone-patellar tendon-bone & 1 \\
Total & 43 \\
\hline
\end{tabular}

TTS: top traction system, TLS: tape locking screw system.

lografts were used in in 4 patients and autologous semitendinosus grafts in 2 patients (tape locking screw system; FH Orthopedics, Heimsbrunn, France) (Table 3).

Thirty-one patients were treated with a single session revision surgery. Seven patients underwent two-session revision where there was some delay until the second session procedure after screw removal. In 5 patients, it was observed the screws had been removed in other hospitals.

\section{Discussion}

Revision ACL surgery is a challenging procedure: postoperative satisfaction rates can be 3-4 times lower than those of primary ACL reconstruction. In this study, we analysed the failure aetiology and the possibilities of revision surgical strategies (graft choice, in particular) with a description of our experience. The causes of ACL reconstruction failure can be divided into three categories: technical errors, biological failure, and traumatic injury. Technical mistakes are usually responsible for reconstruction failure that occurs within 6 months after surgery ${ }^{15)}$. Surgical technique-related errors are the most common cause of relapsing instability after ACL reconstruction, accounting for $77 \%$ to $95 \%$ of all cases of ACL failure. By far the most commonly cited technical error in ACL reconstruction has been non-anatomic tunnel placement, accounting for $70 \%$ to $80 \%$ of all technical failures, with an improperly placed femoral tunnel being the root cause in most cases ${ }^{4,9,16,17)}$. Biological causes include lack of graft incorporation, infection, allograft rejection, and a failure in the ligamentization process ${ }^{15}$. Finally, the incidence of failure resulting from traumatic causes is thought to be around $5 \%$ to $10 \%$. These failure reasons do not exclude each other reciprocally and more than one can contribute to a failure. Before surgery, the patient has to be informed of possible surgical procedures. For example, loss of motion related to the contracture may require surgical debridement in a separate operation or during revision surgery ${ }^{6,7)}$. If the cause is a wrong graft position, this has to be confirmed during the surgical procedure after graft removal. If the whole range of motion is restored, the revision can be done during the same session. A large scar or capsular contracture can be treated more efficiently with a different surgery after a rehabilitation period carried out before revision. The importance of obtaining a complete range of motion before revision must not be underestimated. Wrong tunnel size or placement sometimes necessitates a different surgical session for transplanting some bone in order to fill possible defects, delaying revision for 6-12 weeks ${ }^{6}$.

The choice of graft type for revision surgery is still controversial $^{18)}$. Graft type choice has to be made by the surgeon together with the patient. The most common graft used for primary ACL reconstruction is the homolateral autograft made of the $\mathrm{BPTB}^{19)}$. Therefore, if an autologous tissue is preferred, the options include the gracilis and semitendinosus tendons, quadriceps tendon, or contralateral patellar tendon. Gracilis and semitendinosus tendons are a valid choice for ACL revision surgery ${ }^{1,11,20)}$. Another graft option is represented by allografts, which have both supporters and detractors ${ }^{1,10,21)}$. The advantage of this graft type in revision surgery is the possibility of modelling the bone's oversized blocks when tunnel enlargement or graft and/or fixation system removal causes an osseous loss. In some cases, this can eliminate the need for surgery for drawing of the bone graft. In spite of such advantages, synthetic grafts did not function in a satisfactory way, thus losing consensus on the efficacy. Our graft choice in revision was based on the graft type used in the primary reconstruction, the revision tunnel size, and patient's functional demands. Noyes and Barber-Westin recommend the use of autologous BPTB graft in revision because they think it would result in lower failure rates compared to allograft ${ }^{22)}$. Autograft tissue has been shown to be a good graft option in revision by many authors, with the exception of using previously harvested tissue. Diamantopoulos et al. ${ }^{23)}$, Ferretti et al. $^{24)}$, and Weiler et al. ${ }^{20)}$ have published large case series of patients treated with the use of hamstring autograft in revision surgery. A meta-analysis performed by Freedman et al. ${ }^{2)}$ concluded that patellar tendon autografts were associated with fewer failures and better instrumented laxity in comparison with hamstring tendon autografts but with an increased rate of anterior knee pain. Similar findings were noted in a retrospective series by Barrett et al. ${ }^{25)}$ and Pinczewski et al. ${ }^{26)}$ as well. O'Neill ${ }^{27)}$ 
published a failure rate of $6 \%$ in a series of 48 patients who underwent revision surgery with unharvested ipsilateral grafts (hamstring or BPTB). The results of the ACL revision surgery were not comparable to those obtained from the primary reconstruction $^{28)}$, as we have found in our study and in the literature: the success rate of primary reconstruction is about $92 \%$ compared to $87 \%$ of revision surgery. These less favourable results can be attributed to various causes as in previous operations including meniscus damage or cartilaginous defect. O'Neill ${ }^{27)}$ evaluated 48 revisions with autografts using the IKDC score and achieved good results in $84 \%$ of the cases; $42 \%$ fell into the category $\mathrm{A}$ (normal) and $42 \%$ into the category B (nearly normal). They also studied 38 revisions with cryopreserved allografts: patient satisfaction rate was $84 \%$ and residual anteroposterior laxity (KT-1000; MEDmetric, San Diego, CA, USA) was observed in $84 \%$ of the cases. According to the objective evaluation of the IKDC score, 17 patients were classified as category A, 8 as category $B$, and the remaining 4 as category $\mathrm{C}$, with an average score of 85.56 in the subjective evaluation of the IKDC score.

In our study, $92.2 \%$ of the patients had a normal or a nearly normal knee (IKDC groups A and B). The failure rate was 7.2\%. These results are similar to those documented in recent studies described above.

Limitations of this study include the small number of patients and the retrospective nature of the study that caused difficulty in follow-up of patients who were unavailable for evaluation due to moving or other reasons years after surgery. The significance of this study lies in the fact that it included an extensive analysis of failure aetiologies and options for revision surgical strategies. Understanding of the cause of failure is the first and the foremost important step for successful revision of ACL reconstruction.

\section{Conclusions}

ACL revision surgery is suitable for patients with clinically evident anterior laxity associated with instability during normal daily or sports activities. The aim of the surgery is to stabilize the knee joint, prevent further damage to the cartilage and the menisci, and allow the patient to resume normal daily and/or sports activities.

A successful revision surgery requires accurate preoperative patient evaluation and knee imaging. Then, the surgeon needs to decide the most suitable procedure for each case in terms of graft selection and single/double session surgery and prescribe individualized rehabilitation protocols. It is also important to discuss with the patient before revision about the causes of failure and expectations. Given the complexity of the revision surgery and the possibility of obtaining results inferior to those in primary reconstruction as evidenced in this study. It is necessary to remind the patient that revision should be considered as a rescue procedure. In conclusion, we think that it is fundamental to accurately inform patients of all possible problems before initiation of the revision surgery, even if the procedure is known to yield high rates of excellent and good outcomes.

\section{Conflict of Interest}

No potential conflict of interest relevant to this article was reported.

\section{References}

1. Engelman GH, Carry PM, Hitt KG, Polousky JD, Vidal AF. Comparison of allograft versus autograft anterior cruciate ligament reconstruction graft survival in an active adolescent cohort. Am J Sports Med. 2014;42:2311-8.

2. Freedman KB, D’Amato MJ, Nedeff DD, Kaz A, Bach BR Jr. Arthroscopic anterior cruciate ligament reconstruction: a metaanalysis comparing patellar tendon and hamstring tendon autografts. Am J Sports Med. 2003;31:2-11.

3. Kamath GV, Redfern JC, Greis PE, Burks RT. Revision anterior cruciate ligament reconstruction. Am J Sports Med. 2011;39:199-217.

4. Bach BR, Provencher MT. ACL surgery : how to get it right the first time and what to do if it fails. Thorofare, NJ: SLACK; 2010. p239-51.

5. Shybut TB, Pahk B, Hall G, Meislin RJ, Rokito AS, Rosen J, Jazrawi LM, Sherman OH. Functional outcomes of anterior cruciate ligament reconstruction with tibialis anterior allograft. Bull Hosp Jt Dis (2013). 2013;71:138-43.

6. Adriani E, Summa P, Di Paola B. Pre-operative planning in anterior cruciate ligament reconstruction revision surgery. Joints. 2013;1:25-33.

7. Sahu A, Konarski A, Johnson D. Review of literature: revision anterior cruciate ligament reconstruction. Int J Orthopedic Surg. 2008:11.

8. Allen CR, Giffin JR, Harner CD. Revision anterior cruciate ligament reconstruction. Orthop Clin North Am. 2003;34: 79-98.

9. Wolf RS, Lemak LJ. Revision anterior cruciate ligament reconstruction surgery. J South Orthop Assoc. 2002;11:25-32.

10. OrthopaedicsOne. Revision anterior cruciate ligament (ACL) 
reconstruction [Internet]. Toront, ON: OrthopaedicsOne; 2012 [cited 2016 Nov 18]. Available from: http://www.orthopaedicsone.com/x/qAkCAg.

11. Ahn JH, Lee YS, Ha HC. Comparison of revision surgery with primary anterior cruciate ligament reconstruction and outcome of revision surgery between different graft materials. Am J Sports Med. 2008;36:1889-95.

12. Fox JA, Pierce M, Bojchuk J, Hayden J, Bush-Joseph CA, Bach BR Jr. Revision anterior cruciate ligament reconstruction with nonirradiated fresh-frozen patellar tendon allograft. Arthroscopy. 2004;20:787-94.

13. Steadman JR, Bollom TS. Principles of ACL revision surgery and rehabilitation. Sports Med Arthrosc. 2005;13:53-8.

14. Battaglia TC, Miller MD. Management of bony deficiency in revision anterior cruciate ligament reconstruction using allograft bone dowels: surgical technique. Arthroscopy. 2005; 21:767.

15. Colosimo AJ, Heidt RS Jr, Traub JA, Carlonas RL. Revision anterior cruciate ligament reconstruction with a reharvested ipsilateral patellar tendon. Am J Sports Med. 2001;29:74650.

16. Morgan JA, Dahm D, Levy B, Stuart MJ; MARS Study Group. Femoral tunnel malposition in ACL revision reconstruction. J Knee Surg. 2012;25:361-8.

17. Valis P, Sklensky J, Repko M, Rouchal M, Novak J, Otasevic T. Most frequent causes of autologous graft failure in anterior cruciate ligament replacement. Acta Chir Orthop Traumatol Cech. 2014;81:371-9.

18. Kim HS, Seon JK, Jo AR. Current trends in anterior cruciate ligament reconstruction. Knee Surg Relat Res. 2013;25:16573.

19. Razi M, Sarzaeem MM, Kazemian GH, Najafi F, Najafi MA. Reconstruction of the anterior cruciate ligament: a comparison between bone-patellar tendon-bone grafts and fourstrand hamstring grafts. Med J Islam Repub Iran. 2014;28: 134.
20. Weiler A, Schmeling A, Stohr I, Kaab MJ, Wagner M. Primary versus single-stage revision anterior cruciate ligament reconstruction using autologous hamstring tendon grafts: a prospective matched-group analysis. Am J Sports Med. 2007;35:1643-52.

21. Ferry AT, Van De Velde SK, Li G, Asnis P, Zarins B, Gill TJ. Revision anterior cruciate ligament reconstruction: preoperative planning and technical considerations. Orthop J Harv Med Sch. 2010;12:119-22.

22. Noyes FR, Barber-Westin SD. Revision anterior cruciate surgery with use of bone-patellar tendon-bone autogenous grafts. J Bone Joint Surg Am. 2001;83:1131-43.

23. Diamantopoulos AP, Lorbach O, Paessler HH. Anterior cruciate ligament revision reconstruction: results in 107 patients. Am J Sports Med. 2008;36:851-60.

24. Ferretti A, Conteduca F, Monaco E, De Carli A, D’Arrigo C. Revision anterior cruciate ligament reconstruction with doubled semitendinosus and gracilis tendons and lateral extra-articular reconstruction. J Bone Joint Surg Am. 2006;88: 2373-9.

25. Barrett GR, Noojin FK, Hartzog CW, Nash CR. Reconstruction of the anterior cruciate ligament in females: a comparison of hamstring versus patellar tendon autograft. Arthroscopy. 2002;18:46-54.

26. Pinczewski LA, Deehan DJ, Salmon LJ, Russell VJ, Clingeleffer A. A five-year comparison of patellar tendon versus fourstrand hamstring tendon autograft for arthroscopic reconstruction of the anterior cruciate ligament. Am J Sports Med. 2002;30:523-36.

27. O’Neill DB. Revision arthroscopically assisted anterior cruciate ligament reconstruction with previously unharvested ipsilateral autografts. Am J Sports Med. 2004;32:1833-41.

28. Wright RW, Gill CS, Chen L, Brophy RH, Matava MJ, Smith MV, Mall NA. Outcome of revision anterior cruciate ligament reconstruction: a systematic review. J Bone Joint Surg Am. 2012;94:531-6. 